\title{
10 Schritte zur Schmerzkontrolle
}

\section{Selbstkompetenz bei Kopfschmerz erleichtert das Leiden}

Chronische tägliche Kopfschmerzen (CDH) beeinträchtigen die Lebensqualität - die eigene und manchmal auch jene des Umfelds des Betroffenen. Sie haben auch nicht unbeträchtliche gesellschaftliche und volkswirtschaftliche Kosten zur Folge.

Zwei bis vier Prozent der Nordamerikaner und Europäer leiden täglich an Kopfschmerzen. Unterschiedliche ätiologische Faktoren tragen dazu bei. Die meisten Schmerzen sollen sich mit Hilfe des von Dr. Robert Sheeler von der Mayo Klinik in Rochester vorgestellten 10-Schritte-Plans diagnostizieren und behandeln lassen.

1. Ausschließen lebensbedrohlicher oder progressiver sekundärer Kopfschmerzen

2. Klassifizieren der primären Kopfschmerzen

3. Identifizieren der verschärfenden und erhaltenden Faktoren

4. Identifizieren von Komorbiditäten

5. Prüfen der momentanen akuten Therapie

6. Entgiften bei übermäßigem Medikamentengebrauch

7. Prüfen vergangener präventiver Therapieversuche

8. Erstellen eines Kopfschmerzplans mit akuten, präventiven und LifestyleKomponenten

9. Aufklären des Patienten und Anleitung zum Führen eines Kopfschmerztagebuchs

10. Initiieren eines regelmäßigen Followups zur kontinuierlichen Verbesserung mit dem Ziel, kaum auf Medikation ansprechende, tägliche Kopfschmerzen in kontrollierbare episodische Kopfschmerzen zu verwandeln.

Nach Ausschluss sekundärer Kopfschmerzen können im nächsten Schritt $\mathrm{CDH}$ über die Frequenz und Dauer der Schmerzen klassifiziert werden. Treten diese länger als vier Stunden pro Tag an mehr als 15 Tagen im Monat auf, so fallen sie unter eine der Subkategorien der CDH.

Das Risiko für chronische Kopfschmerzen verstärken Faktoren wie ein erhöhter BMI, Depressionen, ein erhöhter Stresslevel, ein geringer sozioökonomischer Status sowie zu wenig körperliche Aktivität oder Schlaf. So können weniger als sechs und mehr als acht Stunden Schlaf pro Nacht

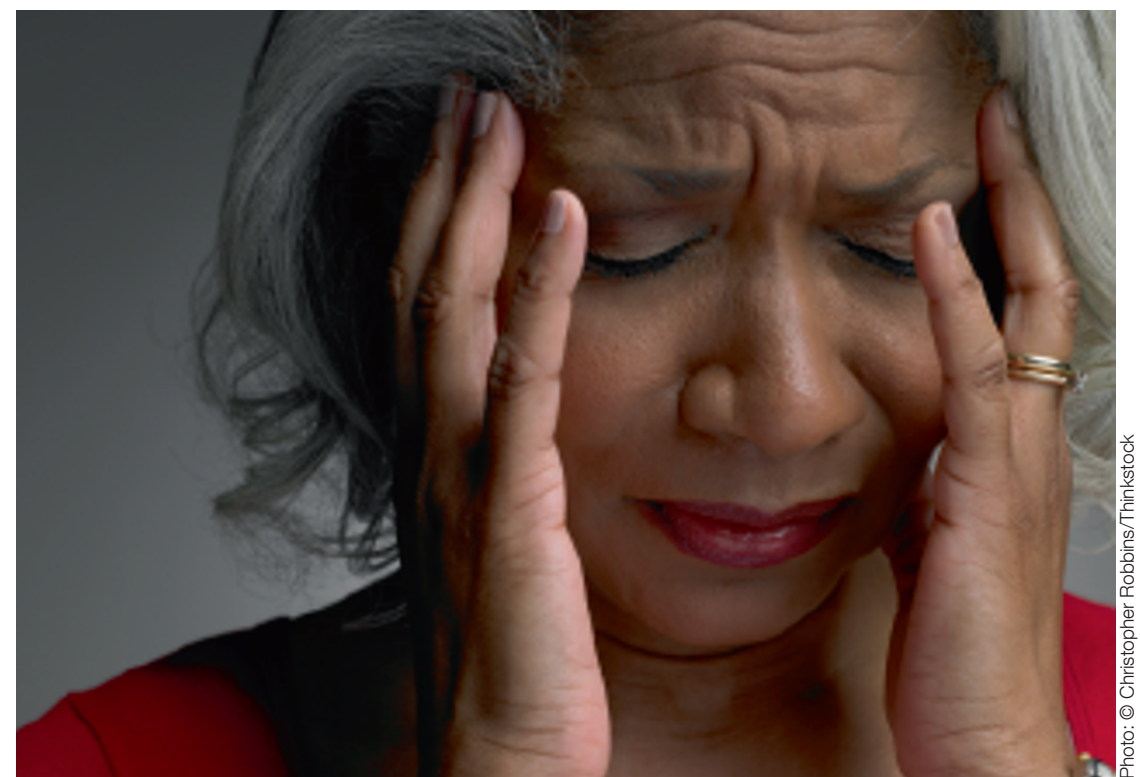

Ein erhöhter Stresslevel fördert Kopfschmerzen.

Kopfschmerzen verschlimmern. Viele Patienten mit chronischen täglichen Kopfschmerzen greifen zu häufig zu Schmerzmitteln, was wiederum „medication overuse headaches" (MOH) bedingen kann. $\mathrm{Zu}$ den riskantesten Präparaten zählen koffeinhaltige, rezeptfreie Medikamente, Barbiturate und Opiate. Deshalb raten Sheeler und seine Mitarbeiter, die aktuelle Medikation des Patienten zu erfragen und bei übermäßigem Medikamentengebrauch eine Entgiftung zu starten. Zur Unterstützung können Therapeuten über kurze Zeit Kortikosteroide, NSAID oder Dihydroergotamin einsetzen.

\section{Buchempfehlung}

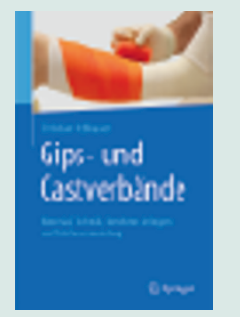

\section{Gips- und Castverbände}

Material, Technik, korrektes Anlegen und Fehlervermeidung

nschließend kann ein individuell au Patienten zugeschnittener Kopfschmerzplan erstellt werden, der präventive sowie akute Therapieoptionen enthält und auch auf die Optimierung des Lebensstils eingeht. Eine enge Zusammenarbeit zwischen Therapeut und Patient sowie eine regelmäßige Kontrolle des Behandlungserfolgs helfen laut Aussage der Autoren dabei, langfristig eine gute Kontrolle über $\mathrm{CDH}$ zu erlangen.

\section{$\ddot{A} Z / j . r$.}

Der Autor beschreibt nach Körperregionen aufgeteilt die einzelnen verfügbaren Verbandstypen, Material, Technik, Gefahren bzw. Fehlermöglichkeiten und Indikationen. Zahlreiche Bilder sowie eine genaue Beschreibung erleichtern dem Anwender das korrekte Anlegen von Gipsverbänden in der täglichen Praxis. 